ORIGINAL ARTICLE

\title{
PI3K inhibition results in enhanced HER signaling and acquired ERK dependency in HER2-overexpressing breast cancer
}

\author{
V Serra ${ }^{1}$, M Scaltriti ${ }^{1,2}$, L Prudkin ${ }^{3}$, PJA Eichhorn ${ }^{1}$, YH Ibrahim ${ }^{1}$, S Chandarlapaty ${ }^{4}$, \\ B Markman ${ }^{1,5}$, O Rodriguez ${ }^{1}$, M Guzman 1 , S Rodriguez ${ }^{3}$, M Gili ${ }^{1}$, M Russillo ${ }^{1}$, JL Parra ${ }^{6}$, \\ S Singh ${ }^{7}, \mathrm{~J}$ Arribas ${ }^{6,8,9}, \mathrm{~N}$ Rosen $^{4}$ and J Baselga ${ }^{1,2,5,8,10}$ \\ ${ }^{1}$ Experimental Therapeutics Program, Vall d'Hebron Institute of Oncology, Barcelona, Spain; ${ }^{2}$ Center for Cancer Research, \\ Massachusetts General Hospital Cancer Center, Boston, MA, USA; ${ }^{3}$ Molecular Pathology Program, Vall d'Hebron Institute \\ of Oncology, Barcelona, Spain; ${ }^{4}$ Program in Molecular Pharmacology and Chemistry and Department of Medicine, Memorial \\ Sloan-Kettering Cancer Center, New York, NY, USA; ${ }^{5}$ Department of Medical Oncology, Vall d'Hebron University Hospital, \\ Barcelona, Spain; ${ }^{6}$ Growth Factors Laboratory, Vall d'Hebron Institute of Oncology, Barcelona, Spain; ${ }^{7}$ Prometheus Therapeutics \\ and Diagnostics, San Diego, CA, USA; ${ }^{8}$ Universitat Autònoma de Barcelona, Barcelona, Spain; ${ }^{9}$ Institució Catalana de Recerca $i$ \\ Estudis Acançats, Barcelona, Spain and ${ }^{10}$ Division of Hematology/Oncology, Massachusetts General Hospital, Boston, MA, USA
}

There is a strong rationale to therapeutically target the phosphatidylinositol 3-kinase/protein kinase $\mathrm{B} / \mathrm{mammalian}$ target of rapamycin (PI3K/AKT/mTOR) pathway in breast cancer since it is highly deregulated in this disease and it also mediates resistance to anti-HER2 therapies. However, initial studies with rapalogs, allosteric inhibitors of mTORC1, have resulted in limited clinical efficacy probably due to the release of a negative regulatory feedback loop that triggers AKT and ERK signaling. Since activation of AKT occurs via PI3K, we decided to explore whether PI3K inhibitors prevent the activation of these compensatory pathways. Using HER2-overexpressing breast cancer cells as a model, we observed that PI3K inhibitors abolished AKT activation. However, PI3K inhibition resulted in a compensatory activation of the ERK signaling pathway. This enhanced ERK signaling occurred as a result of activation of HER family receptors as evidenced by induction of HER receptors dimerization and phosphorylation, increased expression of HER3 and binding of adaptor molecules to HER2 and HER3. The activation of ERK was prevented with either MEK inhibitors or anti-HER2 monoclonal antibodies and tyrosine kinase inhibitors. Combined administration of PI3K inhibitors with either HER2 or MEK inhibitors resulted in decreased proliferation, enhanced cell death and superior anti-tumor activity compared with single agent PI3K inhibitors. Our findings indicate that PI3K inhibition in HER2-overexpressing breast cancer activates a new compensatory pathway that results in ERK dependency. Combined anti-MEK or antiHER2 therapy with PI3K inhibitors may be required in order to achieve optimal efficacy in HER2-overexpressing breast cancer. This approach warrants clinical evaluation. Oncogene (2011) 30, 2547-2557; doi:10.1038/onc.2010.626; published online 31 January 2011

Correspondence: J Baselga, Vall d'Hebron Institute of Oncology (VHIO), Vall d'Hebron University Hospital, Passeig Vall d'Hebron 119-129, E-08035 Barcelona, Spain.

E-mail: jbaselga@vhio.net

Received 5 April 2010; revised and accepted 2 November 2010; published online 31 January 2011
Keywords: PI3K/mTOR; HER2; feedback; ERK; BEZ235

\section{Introduction}

The phosphatidylinositol 3-kinase (PI3K)-protein kinase $\mathrm{B}(\mathrm{PKB} / \mathrm{AKT})$ - mammalian target of rapamycin (mTOR) axis regulates critical cellular functions including metabolism, proliferation, size, survival, migration and angiogenesis (for reviews see Cantley, 2002; Vivanco and Sawyers, 2002; Guertin and Sabatini, 2007). The PI3K/ $\mathrm{AKT} / \mathrm{mTOR}$ pathway is triggered by upstream activation of receptor tyrosine kinases (RTKs) (Yarden and Sliwkowski, 2001). Once activated, these receptors serve as a docking site for PI3K binding, either directly through its regulatory subunit p85 or indirectly via adaptor molecules (that is insulin receptor substrate 1, IRS1).

The PI3K pathway is frequently hyperactivated in breast cancer, as well as in other tumor types, by a number of different mechanisms. First, there is a high frequency of mutations in genes of the PI3K signaling cascade. In breast cancer, PIK3CA (encoding p110- $\alpha$, the catalytic subunit of PI3K) mutations that occur in one third of patients are site specific (located in the helical and kinase domains) and are more frequent in tumors expressing hormone receptors and HER2 (Saal et al., 2005; Kalinsky et al., 2009). Less frequent are PIK3CA amplification and mutations in the AKT gene (Bellacosa et al., 1995; Carpten et al., 2007; LopezKnowles et al., 2010). Second, loss of function of the tumor suppressor phosphatase and tensin homolog is also a common event in breast cancer (Li et al., 1997) and is associated with increased PI3K-pathway activity, metastasis and poor survival (Saal et al., 2005, 2007; Stemke-Hale et al., 2008). And third, HER2 overexpression/amplification is found in $20 \%$ in breast cancer patients (Slamon et al., 1987) and boosts PI3K signaling through HER2/HER3 heterodimerization (Holbro et al., 2003). Taken together, over $70 \%$ of 
breast cancers have a dysregulated PI3K pathway (Lopez-Knowles et al., 2010). Aberrant activation of the PI3K pathway also results in resistance to antiHER2 and other anti-cancer agents (Clark et al., 2002; Nagata et al., 2004; Berns et al., 2007; Eichhorn et al., 2008). Hence, there is a strong rationale to therapeutically target the PI3K/AKT/mTOR axis in breast cancer.

The first available agents for clinical targeting of the $\mathrm{PI} 3 \mathrm{~K} / \mathrm{AKT} / \mathrm{mTOR}$ pathway were allosteric mTORC1 inhibitors (rapamycin analogs, also known as rapalogs). Despite the importance of this pathway in breast cancer, rapalogs have shown only modest efficacy to date (Chan et al., 2005; Baselga et al., 2009; Ellard et al., 2009). A likely explanation for their limited activity may reside in that $\mathrm{mTORC} 1$ inhibition results in the abrogation of an S6K-IRS1-PI3K negative feedback loop, resulting in upstream activation of AKT (O'Reilly et al., 2006; Cloughesy et al., 2008; Tabernero et al., 2008). To overcome this effect, combinations of mTOR inhibitors with agents that target proximal pathway elements such as antibodies against the insulin-like growth factor receptor 1 (IGF-1R) are currently under study. Another potential drawback secondary to mTORC1 inhibition is ERK pathway activation, as evidenced by increased levels of P-ERK in patient tumor samples (Carracedo et al., 2008; Cloughesy et al., 2008). This occurs through the removal of the inhibitory effect of a presumed S6K-PI3K-RAS feedback loop.

The anti-tumor activity of specific class I PI3K or combined PI3K/mTOR inhibitors has been proven in several preclinical models (Fan et al., 2006; Serra et al., 2008; Ihle et al., 2009; Yao et al., 2009) and their clinical efficacy is being evaluated, among other indications, in HER2-overexpressing breast cancer. A potential advantage of PI3K inhibitors over mTORC1 inhibitors would be the prevention of AKT activation, which may limit clinical efficacy of the latter group of compounds. With the intent to study whether PI3K inhibitors prevent this effect, we have found that, although these inhibitors prevent AKT activation, they activate the ERK pathway via an as yet undescribed mechanism, HER2 receptor activation. In the current study, we have characterized this mechanism, as well potential strategies to circumvent it. Our results set the stage for hypothesis-driven combination studies in HER2-positive breast cancer.

\section{Results}

Inhibition of PI3K/AKT/mTOR induces ERK

phosphorylation in HER2-overexpressing cells

Previous findings by our group and others indicate that PI3K and mTOR blockade may result in activation of compensatory pathways that could potentially reduce the anti-tumor effects of $\mathrm{PI} 3 \mathrm{~K} / \mathrm{mTOR}$ inhibitors (O'Reilly et al., 2006; Carracedo et al., 2008; Serra et al., 2008; Courtney et al., 2010). Since PI3K inhibitors are currently being investigated in HER2-overexpressing breast cancer due to their frequently aberrant PI3K activation status, we decided to investigate further the presence of compensatory pathways in this tumor type.
To this end, we treated several HER2-overexpressing breast cancer cell lines with BEZ235, an imidazoquinoline that inhibits class I PI3K catalytic activity by competing at its ATP-binding site and that also inhibits mTOR catalytic activity (Maira et al., 2008). Therapy with BEZ235 resulted in a dose-dependent increase in ERK phosphorylation in all studied cells (BT474 and MCF7HER2, Figure 1a; SKBR3, Supplementary Figure 1; and MDA-MB-361, data not shown). The phosphorylation of p90RSK, a downstream effector dependent of ERK signaling, also increased following BEZ235 exposure. In HER2 negative cell lines such as parental MCF7 the activation of P-ERK upon PI3K/mTOR inhibition was less pronounced. In MDA-MB-468 and MDA-MB-231 cells, we could not detect an increase in P-ERK, probably due to inherent high signaling of P-ERK in these cells (EGFR overexpressing and K-RAS mutated cells, respectively) (Supplementary Figure 2).

BEZ235 at low concentrations $(<100 \mathrm{~nm})$ has a predominant mTORC1 inhibitory activity whereas at relatively high concentrations $(\approx 500 \mathrm{nM})$ it is a dual $\mathrm{PI} 3 \mathrm{~K} / \mathrm{mTORC} 1 / 2$ inhibitor (Serra et al., 2008). This concentration-dependent effect of BEZ235 explains the increased phosphorylation of AKT at both Ser473 and Thr308 seen at lower concentrations of BEZ235 $(\approx 25 \mathrm{~nm})$ in cell lines with low basal levels of P-AKT (MCF7-HER2, Figure 1a; SKBR3, Supplementary Figure 1; and MDA-MB-361, data not shown). On the other hand, at higher BEZ235 concentrations (500 nM) there was complete inhibition of P-AKT (Figure 1a). The observed inhibition of phosphorylated S6 served as readout of BEZ235 mTORC1 activity.

To determine whether activation of ERK was a consequence of pharmacological blockade of the PI3K/ mTORC pathway rather that a specific effect of BEZ235, we studied a number of different anti-PI3K/ mTOR agents. ERK activation was observed with all studied agents including a pan-PI3K inhibitor (GDC0941), a p110- $\alpha$ inhibitor (PIK-90), the already mentioned dual p110/mTOR inhibitor BEZ235, an AKT1/2/ 3 allosteric inhibitor (MK-2206), an allosteric mTOR inhibitor (RAD001) and an mTORC1/2 catalytic inhibitor (Torin1) (see MCF7-HER2, Figure 1b; and BT474, data not shown). Although the increase in ERK phosphorylation by mTORC1 allosteric inhibitors has been associated with active PI3K (Carracedo et al., 2008), we observed a measurable increase in HER2 phoshorylation, which suggests co-existence of two mechanisms.

In an attempt to dissect the potential source of HER2 phosphorylation, we observed that AKT is inhibited by all the compounds with the exception of RAD001, an allosteric inhibitor of $\mathrm{mTORC1}$, for which the increase of P-AKT was expected (O'Reilly et al., 2006). Regarding the different patterns of inhibition of S6 phosphorylation, we observed that the inhibitors of the upper pathway (the PI3K inhibitors GDC-0941 and PIK-90, and the allosteric AKT1/2/3 inhibitor) partly inhibit mTORC1 activity and therefore only a modest decrease of P-S6 is observed (similar data for P-4EBP1, not shown). On the other hand, inhibitors that act further 

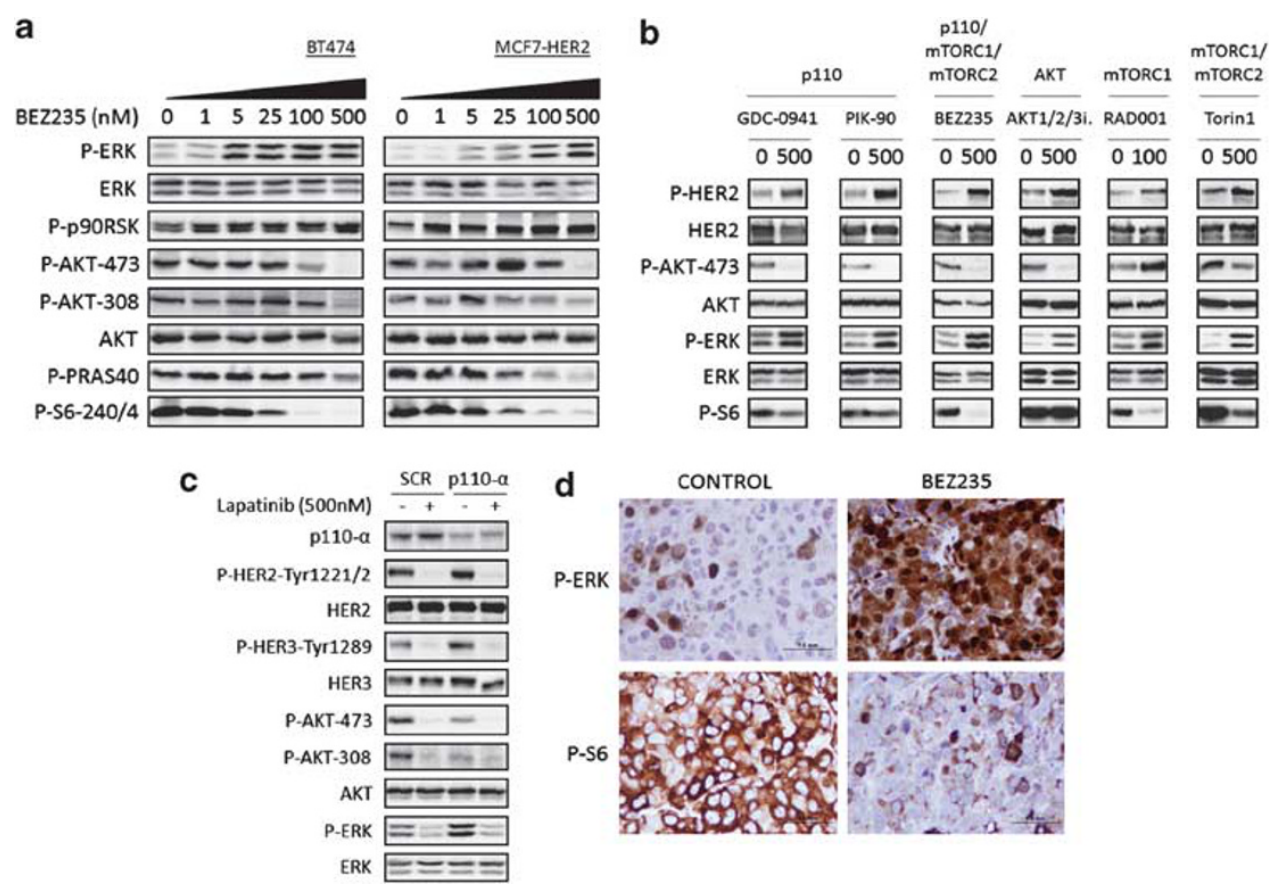

Figure 1 PI3K-pathway inhibition induces ERK phosphorylation in HER2-overexpressing cells. (a) Immunoblots of BT474 and MCF7-HER2 treated with BEZ235 (0-500 nM) for 24 h. (b) Immunoblots of MCF7-HER2 cells treated for $24 \mathrm{~h}$ with different PI3Kpathway inhibitors at the indicated concentrations (all in nM). (c) Immunoblots of p110- $\alpha$ knockdown MCF7-HER2 cells. SCR, scramble control; p110- $\alpha$, siRNA for p110- $\alpha$. Lapatinib treatment for $24 \mathrm{~h}$. (d) Immunohistochemistry (IHC) of BT474-trastuzumab resistant (BT474-Tr) xenografts treated with BEZ235 $(40 \mathrm{mg} / \mathrm{kg}, 4 \times \mathrm{QD})$ with the indicated phospho-proteins. IHC pictures are representative of the 6-h time point.

downstream in the pathway and target directly mTORC1 (BEZ235, RAD001, Torin1) are more efficient in decreasing the levels of P-S6 (and P-4EBP1, not shown). Therefore, the common pattern across all inhibitors and potential source of HER2 phosphorylation was AKT blockade (with the exception of RAD001 as already mentioned).

The observed ERK activation with multiple agents suggests a class-effect. To rule out pharmacological off target effects of these compounds, we knocked down the expression of $p 110-\alpha$ by means of siRNA and observed increase phosphorylated HER2, HER3 and ERK (Figure 1c). The knockdown of Akt1/2/3 reproduced similar results (data not shown). Activation of ERK as well as a decrease in P-S6 following BEZ235 treatment was observed also in vivo in human tumor xenografts (BT474-Tr, Figure 1d and Supplementary Figure 3a; BT474 and MDA-MB-361, data not shown) and in mouse skin (Supplementary Figure $3 b$ ). It is worth noting that activation of ERK is not an immediate event and that it was detected only $6 \mathrm{~h}$ after compound administration in our in vivo experiments (Supplementary Figure 3a). This delay in activation was also observed in vitro (data not shown) and could have implications for when to monitor ERK activation in clinical trials.

PI3K/mTOR inhibition induces HER receptor activation Since in other model systems we had previously shown that activation of compensatory pathways with mTORC1 inhibitors occurred via activation of the
RTK IGF-1R signaling (Shi et al., 2005; O’Reilly et al., 2006), we decided to investigate whether increased ERK phosphorylation following PI3K/mTOR inhibition in HER2-positive cells was also accompanied by activation of RTKs.

We used a phospho-RTK array to study the effects of BEZ235 administration on the activation status of 42 RTKs, including HER receptors, IGF-1R, MET and PDGFR. The main finding of this experiment was a pronounced increase in phosphorylation of members of the HER family. With longer exposure of the phosphoRTK array, activation of other receptors (IGF-1R, EphA7) was detected, albeit only very modestly (data not shown). In BT474 cells, there was an increase of both P-HER2 and P-HER3 (Figures 2a and b). These results were confirmed in MCF7-HER2 cells (Figure 2b) in which we also detected an increased EGFR phosphorylation. The PI3K inhibitor-mediated activation of HER receptors was prevented by the anti-HER2 tyrosine kinase inhibitor (TKI) lapatinib. Beside HER receptor activation, we also detected an increase of total HER3 (more marked in BT474 cells) and total EGFR (more marked in MCF7-HER2 cells) levels in cells treated with BEZ235 alone or in combination with lapatinib (see Figure 2b). This increase in HER3 protein was associated with enhanced transcription of mRNA for this receptor (Figure 2c), suggesting that PI3K negatively regulates receptor expression and that this negative feedback is released with PI3K inhibition (Makhija et al., 2010). To a lesser extent, HER2 transcription also increased in the same conditions. No significant differences (we 


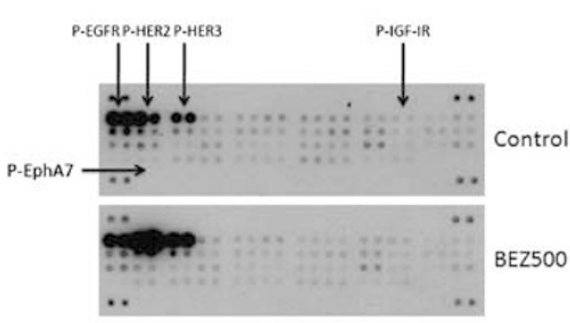

c

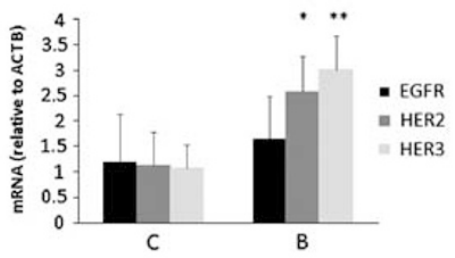

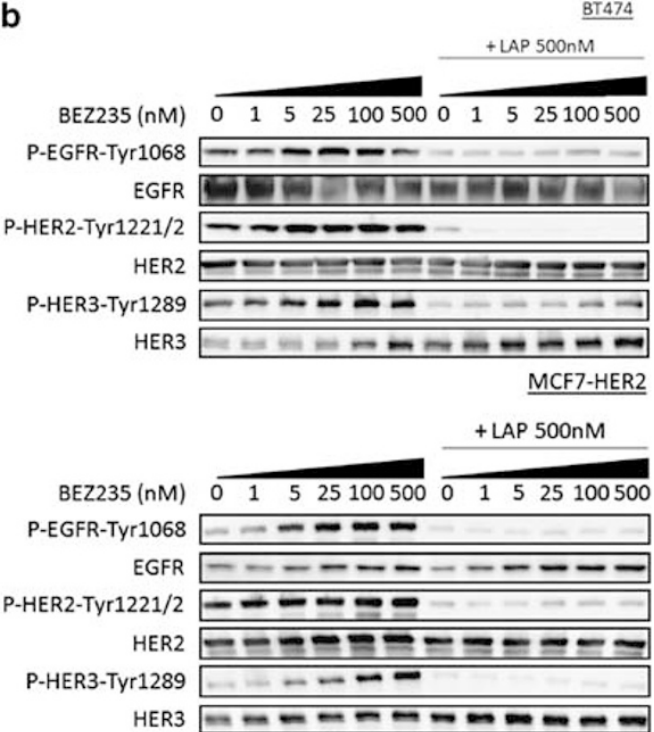

Figure 2 PI3K-pathway inhibition induces HER receptors expression and phosphorylation in HER2-overexpressing cells. (a) Phospho-RTK Array of cell lysates from BT474 cells treated with either DMSO (Control) or $500 \mathrm{~nm} \mathrm{BEZ235} \mathrm{(BEZ500)} \mathrm{for} 24 \mathrm{~h}$. The array contains 42 duplicated spots for specific P-RTKs as well as positive and negative controls. (b) Immunoblots of protein lysates from BT474 and MCF7-HER2 cells treated with either BEZ235 alone $(0-500 \mathrm{nM})$ or the combination of BEZ235 $(0-500 \mathrm{nM})$ and the tyrosine kinase inhibitor lapatinib (LAP, $500 \mathrm{~nm}$ ) for $24 \mathrm{~h}$. (c) EGFR, HER2 and HER3 mRNA levels (related to $\beta$ actin, ACTB) measured by qRT-PCR. BT474 cells were treated with $500 \mathrm{~nm}$ BEZ235 for $24 \mathrm{~h}$. ${ }^{*} P<0.05$; ${ }^{* *} P<0.01$.

acknowledge a trend though) in EGFR mRNA levels were found in BT474 cells treated with BEZ235 (Figure 2c). Because FoxO transcription factors can modulate the expression of HER3 (Garrett et al., 2009; Chandarlapaty et al., 2011), we confirmed that PI3K, AKT and dual PI3K/mTOR inhibitors enhanced FoxO3a nuclear localization along with an increase of HER3 protein (Supplementary Figure $4 \mathrm{a}$ and $\mathrm{b}$ and Chandarlapaty et al., 2011).

\section{PISK inhibition induces HER $2 / 3$ receptor dimerization and increases binding of the adaptor GRB2 to HER2 and the p85 subunit of PI3K to HER3}

We attempted next to dissect the mechanism responsible for HER receptors activation and consequent ERK phosphorylation following $\mathrm{PI} 3 \mathrm{~K} / \mathrm{mTOR}$ inhibition. Increased HER2/HER3 dimer formation was observed upon BEZ235 treatment as shown by cross-linking of membrane proteins followed by immunoprecipitation with an anti-HER2 antibody (Figure 3a). This effect was not exclusive for HER2/HER3 dimers since we could also detect increased HER2/EGFR dimer formation in SKBR3 cells, which express more EGFR compared with BT474 cells (data not shown). However, in cells with low levels of EGFR such as MCF7-HER2 cells, we could not detect membrane EGFR dimerization to HER2 or HER3 following treatment with BEZ235 (Supplementary Figure 5a).

HER receptor dimerization initiates downstream signaling via recruitment of both adaptor molecules and the p 85 regulatory subunit of PI3K (Olayioye et al., 2000; Citri and Yarden, 2006; Baselga and Swain, 2009). Accordingly, in our experimental model we observed an increased binding of the adaptor molecule GRB2 and $\mathrm{p} 85$ to the HER receptors, responsible for ERK and AKT activation, respectively. There was a dosedependent increase in the binding between HER2 and GRB2 in MCF-7HER2 cells treated with BEZ235 (Figure 3b, upper panel). The same result was reproduced in BT474 and SKBR3 cells (data not shown). Similarly, we observed enhanced binding between HER3 and p85 upon BEZ235 treatment (Figure 3b, lower panel). Importantly, the increase of HER2/GRB2 and HER3/p85 binding was prevented by the HER2 TKI lapatinib (Figure $3 b$ ). We could not detect an increase in binding between EGFR and GRB2, ruling out a possible contribution of increased P-EGFR to P-ERK in this model (Supplementary Figure 5b).

\section{ERK activation is prevented by HER2 and MEK1/2 inhibitors}

In order to confirm that ERK activation following PI3K/mTOR inhibition occurs via HER receptor activation, we treated both BT474 and MCF7-HER2 cells with the HER2 TKI lapatinib and the anti-HER2 monoclonal antibody trastuzumab (Figures $4 \mathrm{a}$ and $\mathrm{b}$ ). Both agents inhibited ERK activation, with a greater inhibition by lapatinib, a more potent receptor signaling inhibitor than trastuzumab (Scaltriti et al., 2009).

An alternative strategy to block ERK phosphorylation is to use MEK inhibitors, given that MEK is immediately upstream from ERK. The MEK1/2 inhibitor AZD6244 completely abolished the BEZ235induced phosphorylation of ERK (Figure 4c). As previously described (Mirzoeva et al., 2009), MEK $1 / 2$ inhibition results in AKT phosphorylation. Similar results were obtained using SKBR3 cells treated with 


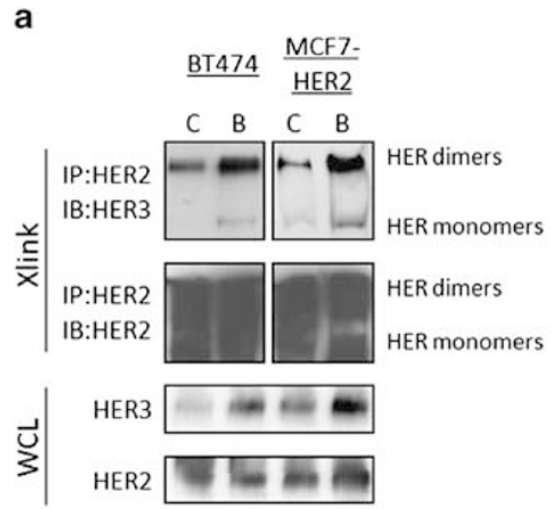

b

MCF7-HER2

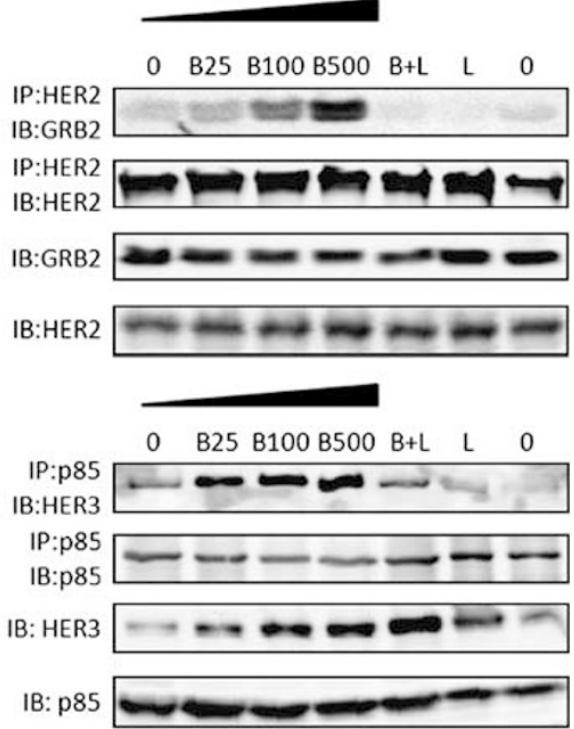

Figure 3 BEZ235 increases HER2/HER3 heterodimerization and receptor signaling. (a) Immunoblots of lysates from BT474 and MCF7-HER2 cells treated with DMSO (c) or BEZ235 (b, $500 \mathrm{~nm}$ ) for $24 \mathrm{~h}$ and subjected to membrane cross-link (Xlink). Cross-linked protein lysates were immunoprecipitated with (IP) and immunoblotted (IB) against the indicated antibodies. Whole-cell lysate (WCL) serve as input controls. (b) Immunoblots of lysates from MCF7-HER2 cells treated with BEZ235 (b, 0-500 nM), lapatinib (L, 500 nM) or the combination (B + L, both at $500 \mathrm{~nm}$ ) for $24 \mathrm{~h}$. Total lysates were immunoprecipitated with (IP) and immunoblotted (IB) against the indicated antibodies.
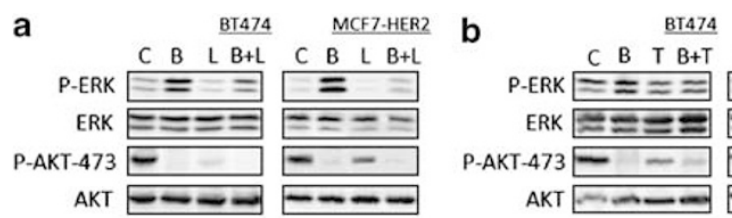

BT474

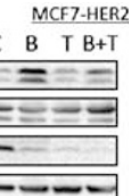

C

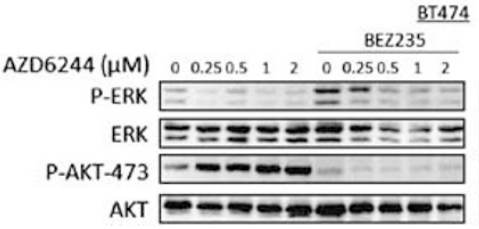

T474

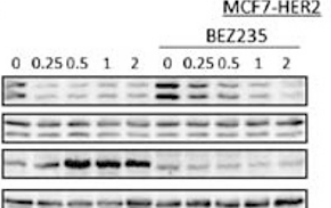

d

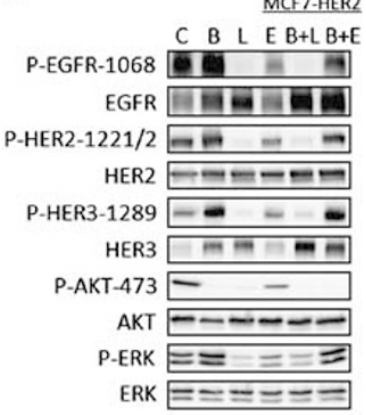

Figure 4 BEZ235-induced P-ERK pathway is prevented with agents inhibiting HER2 and MEK1/2 activity. (a) Immunoblots of lysates from BT474 and MCF7-HER2 cells treated with DMSO (c), BEZ235 (B, 500 nM) and lapatinib (L, 500 nm) or the combination of both inhibitors for $24 \mathrm{~h}$. (b) Immunoblots of lysates from BT474 and MCF7-HER2 cells treated with DMSO (c), trastuzumab (T, $50 \mathrm{nM}, 72 \mathrm{~h}$ ) and BEZ235 (B, $100 \mathrm{~nm}, 24 \mathrm{~h}$ ) or the combination of both. (c) Immunoblots of lysates from BT474 and MCF7-HER2 cells treated with AZD6244 and BEZ235 used at the indicated concentrations for $24 \mathrm{~h}$. (d) Immunoblots of MCF7-HER2 cells treated with DMSO (c), BEZ235 (B, $500 \mathrm{~nm}$ ) and lapatinib (L, $500 \mathrm{~nm}$ ) as well as with erlotinib (E, $500 \mathrm{~nm}$ ) and the indicated combinations for $24 \mathrm{~h}$. All protein lysates were immunoblotted with the indicated antibodies.

BEZ235 combined with lapatinib, trastuzumab or AZD6244 (data not shown). To rule out a possible contribution of activated EGFR, we assessed whether erlotinib, a TKI with selectivity for EGFR (Figure 4d), could prevent the BEZ235-induced increase of P-ERK. At $500 \mathrm{~nm}$, erlotinib markedly reduced P-EGFR without affecting BEZ235-dependent P-ERK activation.

Although we did not detect a significant increased activation of other RTKs with BEZ235 administration (see above), we wanted to further exclude the possibility that other receptors (besides the HER family) were involved in this process. We tested whether the IGF-1R inhibitor AEW541 and the SRC-kinase inhibitor-I
(SRC-KI-I) could prevent ERK transactivation. These two tyrosine kinases have been previously described to have a role in the activation of AKT and ERK by rapalogs (O'Reilly et al., 2006; Chaturvedi et al., 2009). We found that they did not prevent ERK activation following treatment with BEZ235 (Supplementary Figure 6). As a positive control, we used UO126, an additional MEK $1 / 2$ inhibitor that, in a similar manner as AZD6244, also showed complete inhibition of ERK. Importantly, HER2 phosphorylation was not affected by MEK inhibition further corroborating that RTKs activation occurs independent from ERK activation (Supplementary Figure 6). 
HER2 blockade potentiates anti-proliferative and pro-apoptotic effects of PI3K/mTOR inhibition

The compensatory activation of HER receptors and the ERK pathway could serve as an escape mechanism to PI3K blockade and diminish the anti-proliferative and proapoptotic potential of PI3K inhibitors. For this reason, we asked whether dual blockade of HER2 (or MEK1/2) and $\mathrm{PI} 3 \mathrm{~K} / \mathrm{mTOR}$ would be superior to single inhibition of $\mathrm{PI} 3 \mathrm{~K} / \mathrm{mTOR}$ in reducing cell proliferation and/or promoting cell death. We found that the combination of BEZ235 with lapatinib, trastuzumab or AZD6244 was more effective than single agents in reducing cell proliferation (Figure 5a). Similar results were obtained at higher doses of BEZ235 (500 nM, targeting both PI3K/mTOR) and shorter time points (5 days, data not shown). We also observed that the same combinations of agents enhanced the apoptosis induced by BEZ235 alone (Figure $5 \mathrm{~b}$ and data not shown). Of all the evaluated combinations, BEZ235 with lapatinib showed the highest anti-proliferative and pro-apoptotic activity.

\section{HER 2 or $M E K 1 / 2$ inhibition potentiates the in vivo}

BEZ235 anti-tumor activity

We then measured the activity of anti-HER2 therapy or MEK1/2 inhibition in combination with BEZ235 in reducing tumor growth of BT474-Tr xenografts. We first attempted the combination of BEZ235 and lapatinib but, even at suboptimal doses and with wide intervals of administration of the two compounds (morning BEZ235/afternoon lapatinib), it resulted in

unacceptable toxicity (body weight loss $>10 \%$, dehydration, lethargy) in two different mouse strains (Hsd:Athymic Nude-Foxn $1^{m u}$ and Crl:NU-Foxn $1^{m u}$ ) on a continuous dose regime. Whether this implies that the combination will result in excessive toxicity in humans is unknown but our findings should serve as a cautionary note. The combination of BEZ235 and trastuzumab was non-toxic and resulted in a significantly improved tumor growth inhibition compared with either agent alone (Figure 6a, left panel).

Using the same BT474-Tr model, we determined the anti-tumor activity of the MEK1/2 inhibitor AZD6244 in combination with BEZ235. As previously described, MEK1/2 inhibition alone has little anti-tumor activity in BT474-derived tumors (Solit et al., 2006; Hoeflich et al., 2009). However, AZD6244 in combination with BEZ235 resulted in a significant reduction of tumor growth compared with either agent used as monotherapy (Figure 6a, right panel). This increased anti-tumor activity was accompanied by a decreased in vivo proliferation as measured by reduced Ki67 staining (Supplementary Figure 7).

In vivo pharmacodynamic assessment of ERK phosphorylation showed that trastuzumab, lapatinib and AZD6244 all prevented BEZ235-induced ERK phosphorylation, abeit with differing potencies (Figures $6 \mathrm{~b}$ and c). As single agents, lapatinib, AZD6244 and trastuzumab reduced the levels of P-ERK when compared with control xenografts whereas P-S6 expression (a read out of PI3K/mTOR inhibition) was
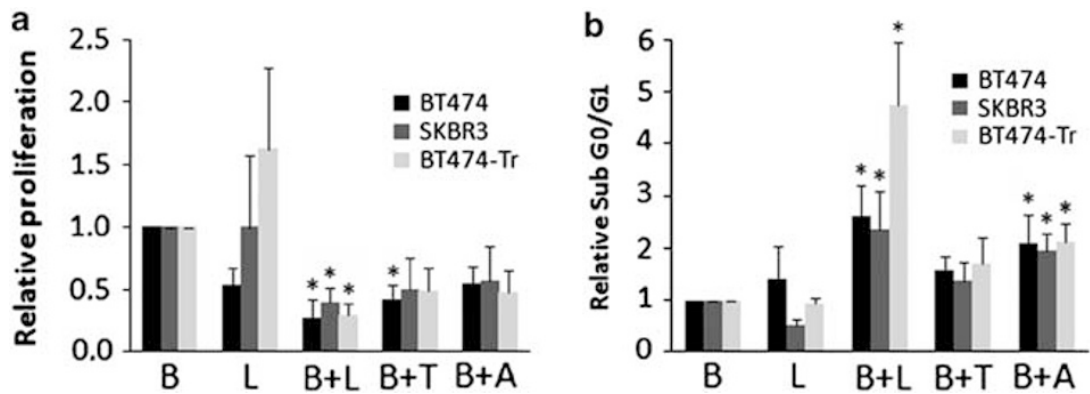

Figure 5 The combination of BEZ235 with either anti-HER2 agents or an MEK inhibitor reduces cell proliferation and induces apoptosis. (a) Cell proliferation assay of BT474, SKBR3 and BT474-Tr cells treated with BEZ235 (B, $10 \mathrm{~nm}$ ), lapatinib (L, 20 nM), trastuzumab (T, $1 \mathrm{nM})$, AZD6244 (A, $2 \mu \mathrm{M})$ or the indicated combinations for 15 days. Cell proliferation was quantified by crystal violet. The fold difference of viable cells is shown relative to the viable cells treated with BEZ235. In order to better visualize the differences between BEZ235-treated cells as single agent or in combination with the aforementioned agents, proliferation of control, trastuzumab and AZD6244-treated cells has been omitted. (b) Cell death expressed as sub-G0/G1 of BT474, SKBR3 and BT474-Tr cells treated with BEZ235 (B, $100 \mathrm{~nm})$, lapatinib (L, $100 \mathrm{~nm})$, trastuzumab (T, $50 \mathrm{~nm})$, AZD6244 (A, $2 \mu \mathrm{M})$ or the indicated combinations for $72 \mathrm{~h}$. The subG0/G1 population was quantified by FACS analysis and related to the amount of apoptotic cells upon BEZ235 treatment. Error bars indicate SE of two (a) or three (b) independent experiments. $* P<0.05$.

Figure 6 Combined inhibition of PI3K/mTOR and HER2 or PI3K/mTOR and MEK results in greater inhibition of tumor growth and prevents ERK transactivation in vivo. (a) Left panel: tumor growth of BT474-Tr-derived xenografts treated with placebo (c), BEZ235 (B, $20 \mathrm{mg} / \mathrm{kg}, \mathrm{QD})$, trastuzumab (T, $20 \mathrm{mg} / \mathrm{kg}$, BIW) or the combination of both. Right panel: tumor growth of BT474-Tr xenografts treated with placebo (c), BEZ235 (B, $25 \mathrm{mg} / \mathrm{kg}$, QD) as single agent or combined with AZD6244 (A, $8 \mathrm{mg} / \mathrm{kg}$, QD). ${ }^{*} P<0.05$. Error bars indicate SE of at least 10 mice. (b) Immunofluorescence of BT474-Tr xenografts treated with placebo (c), BEZ235 $(40 \mathrm{mg} / \mathrm{kg}, 4 \times \mathrm{QD})$, lapatinib $(120 \mathrm{mg} / \mathrm{kg}, 3 \times \mathrm{QD})$, trastuzumab $(20 \mathrm{mg} / \mathrm{kg}$, days 1 and 3$)$, AZD6244 $(15 \mathrm{mg} / \mathrm{kg}, 4 \times \mathrm{QD})$ or in combination as indicated. Red indicates primary antibodies; blue indicates DAPI staining. White bars, $200 \mu \mathrm{m}$. (c) Images were quantified with AQUA ${ }^{\mathrm{TM}}$. Red indicates nuclear stain; blue indicates cytoplasmic stain. $* P<0.05 ; * * P<0.01$. (d) Cartoon outlining the $\mathrm{PI} 3 \mathrm{~K} / \mathrm{AKT} / \mathrm{mTOR}$ and RAS/RAF/MEK/ERK pathways. Inhibition of PI3K/AKT causes nuclear relocalization of the FoxO3a transcription factor and increase of HER 3 mRNA and protein. The augmented HER3 is able to heterodimerize with HER2, therefore enhancing receptor phosphorylation and signaling to the PI3K and ERK pathways. 
inhibited only by BEZ235 and lapatinib. We could also detect increased P-HER2 induced by BEZ235 treatment in vivo along with reduced P-AKT (Supplementary
Figure 8a). These findings were validated using the Collaborative Proximity ImmunoAssay technique (channel enhanced enzyme reaction immunoassay) to

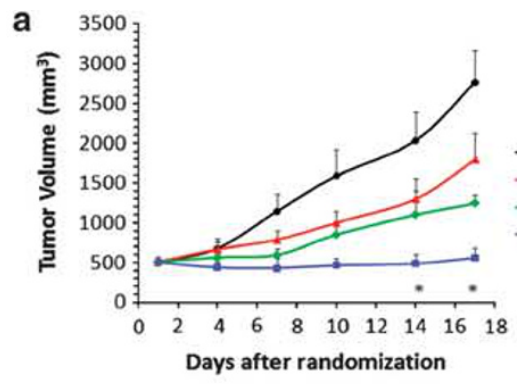

b
P-ERK

BEZ235

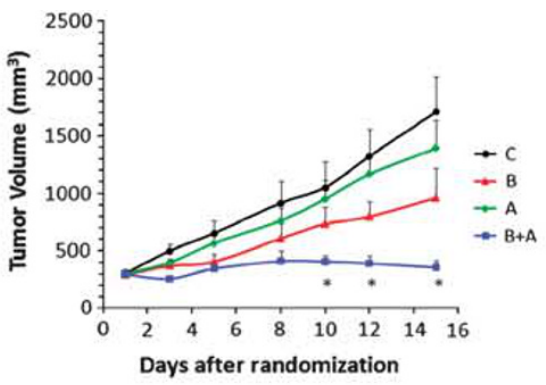

P-S6

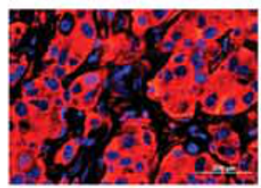

BEZ235
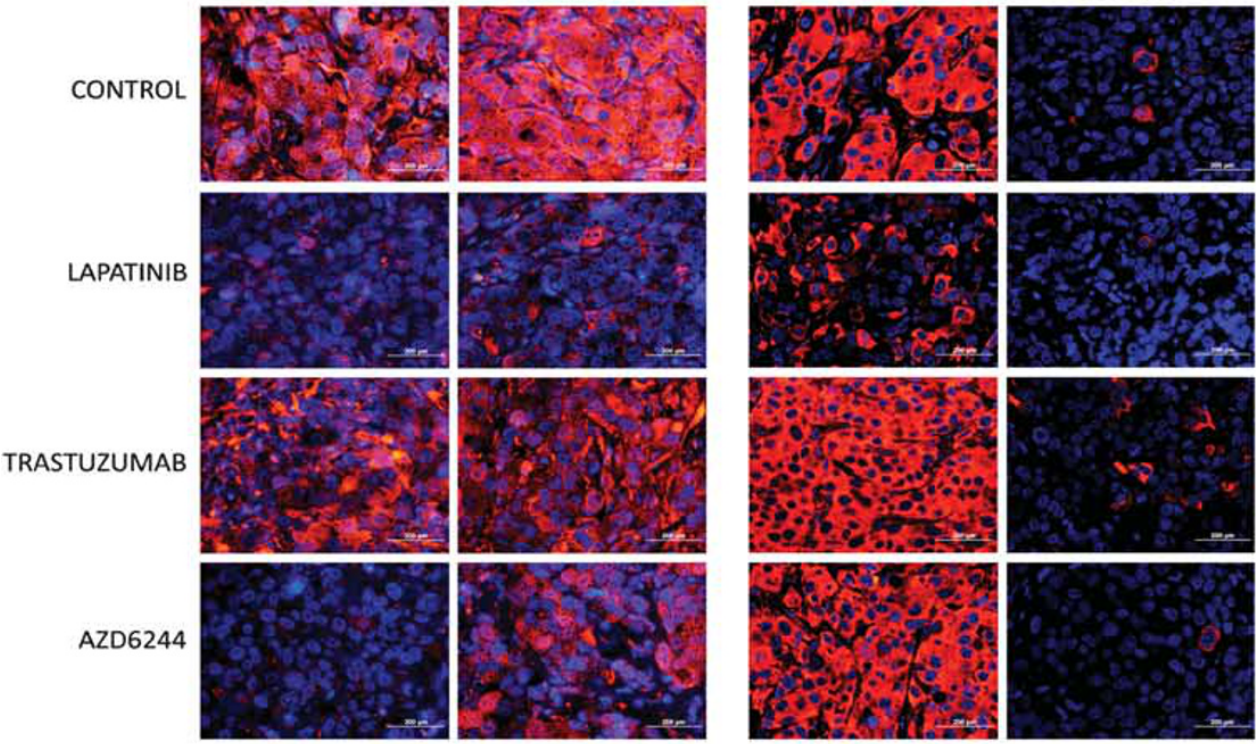

c
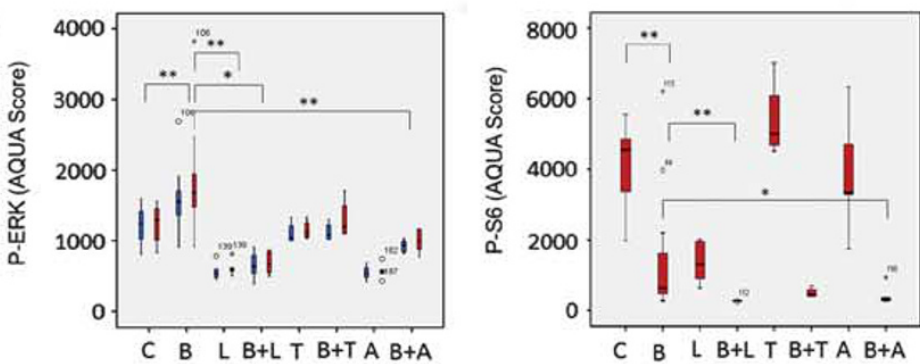

d

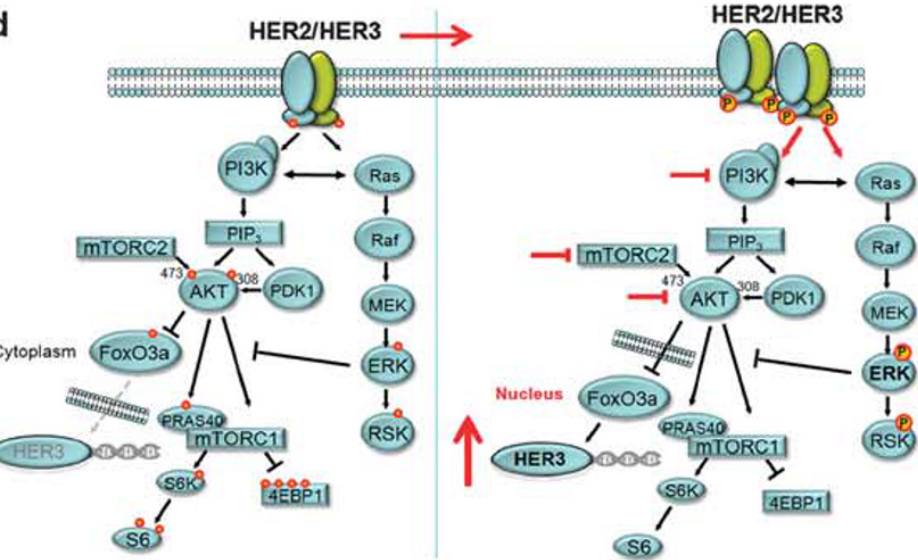


quantify the phosphorylation status of HER2 and AKT in the available spare paired frozen samples, showing a similar trend to the immunohistochemistry results (Supplementary Figure 8b).

\section{Discussion}

In the present study, we have shown that, in HER2positive breast cancer models, the inhibition of the PI3K/ AKT/mTOR pathway results in a compensatory activation of the ERK signaling pathway. This enhanced ERK signaling occurs as a result of activation of HER family receptors as evidenced by increased expression of HER3, induction of HER receptors dimerization and phosphorylation and binding of adaptor molecules to HER2 and HER3. Enhanced HER3 protein was observed independently of HER2 overexpression and is due to transcriptional regulation via FoxO transcription factors (Figure 6d; Garrett et al., 2009; Chandarlapaty et al., 2011), which are activated upon AKT-mediated nuclear relocalization (Brunet et al., 1999). Allosteric inhibition of mTORC1 lead to a milder increase in HER2 and HER3 phosphorylation compared with the other PI3Kpathway inhibitors, which was uncoupled to an increase in total HER3 protein and FoxO3a nuclear translocation (data not shown). This may indicate that P-ERK activation following mTORC1 inhibition occurs mainly via the PI3K-RAS signaling (Carracedo et al., 2008).

Further evidence that enhanced HER2 signaling is responsible for the observed ERK activation is provided by the observation that HER2 inhibitors prevented ERK activation. On the contrary, small molecule kinase inhibitors of EGFR, IGF-1R or SRC failed to reverse ERK activation secondary to BEZ235 treatment. Taken together, our findings suggest that PI3K inhibition in HER2-overexpressing breast cancer results in hyperactivation of ERK that could potentially result in decreased efficacy of PI3K inhibitors. Anti-HER2 and MEK inhibitors did not only abolish ERK phosphorylation but also increased the anti-proliferative and proapoptotic effects of PI3K inhibitors. As a result of our observations, we would propose that a preferred therapeutic strategy in HER2-overexpressing breast cancer would be the administration of PI3K inhibitors in combination with either anti-HER2 agents or MEK inhibitors, instead of PI3K inhibitors given alone.

Our findings provide additional evidence that in cancer cells with constitutive PI3K activation, negative regulatory feedback loops silence compensatory pathways (that is RAS/RAF/ERK) and maintain the dependency on PI3K/AKT/mTOR signaling, a defining feature of oncogene addiction. However, upon pathway (or oncogene) blockade, these inhibitory loops are released and compensatory pathways are activated. We are also learning that these compensatory feedback loops are present at multiple levels of the pathway. Subsequently, different pathways may awake from a 'dormant state' depending on the level at which the therapeutic intervention occurs. For example, we initially reported that mTORC1 inhibition results in activation of PI3K that resulted in ERK activation (Carracedo et al., 2008). Here, we show that with direct PI3K blockade another mechanism comes to play, such as ERK activation via RTKs. While activation of RTKs also occurs with mTORC1 allosteric inhibitors, it is less prominent than with PI3K inhibitors. The type of RTK being activated may also vary in a tumor-dependent context since we have previously shown activation of IGF-1R signaling with $\mathrm{mTORC1}$ inhibitors in nonHER2-overexpressing breast cancer cells (O'Reilly et al., 2006; Tabernero et al., 2008). In contrast, in HER2overexpressing cancer cells, HER receptors signaling appears to be responsible for ERK activation. Our findings have implications for future therapeutic strategies, where the selection of drug combinations will depend on both the type of PI3K/AKT/mTOR inhibitor as well as the cancer type. For example, based on the increase of IGF-1R signaling observed with the mTORC1 inhibitors, we are now conducting a clinical trial combining an mTORC1 inhibitor with a monoclonal antibody that inhibits IGF-1R signaling in nonHER2-overexpressing breast cancer. This combination would be less appealing in HER2-positive breast cancers treated with a PI3K inhibitor.

Our study also highlights the role of the RAS/RAF/ MEK/ERK pathway as an escape mechanism to PI3K blockade. The ERK cascade is at the heart of signaling networks that govern proliferation, differentiation and cell survival (Kolch, 2000). RAS is activated by RTKs via the adaptor molecule GRB2 and subsequently signals through RAF, RALGDS or PI3K itself. This pathway is frequently activated in human tumors, often through gain-of-function mutations of the RAS and RAF family members (Davies et al., 2002). Tumors with mutated BRAF, such as melanoma, are exquisitely sensitive to selective V600-BRAF and MEK inhibitors (Solit et al., 2006; Flaherty et al., 2009). Mutations in this pathway may lead to therapeutic resistance to PI3K inhibitors that is reverted by MEK inhibitors. For example, mouse models of mutated KRAS lung cancer are resistant to BEZ235, but combining BEZ235 with an MEK inhibitor results in synergistic tumor shrinkage (Engelman et al., 2008). In breast cancer, BRAF and RAS mutations are rare, which has supported the use of PI3K inhibitors as single agents. However, BRAF or RAS mutations may not be required for resistance to PI3K inhibitors. For example, resistance to PI3K inhibitors has been described in a subset of basal-like breast cancer cells that have a RAS-driven signature (Hoeflich et al., 2009). In the present study of HER2positive breast cancer cells that lack RAS/RAF mutations or a RAS-driven signature, HER2 activation appears to be a novel mechanism of acquired 'ERK dependence'. As in the case of RAS mutant tumors and tumors with a RAS-driven signature, the addition of an MEK inhibitor should be beneficial in this setting. Our studies confirm this since the addition of an MEK inhibitor (or prevention of pathway activation with antiHER2 agents) to PI3K inhibitors results in greater inhibition of proliferation, augmented apoptosis and the significant tumor shrinkage observed in vivo. 
In conclusion, our data support the use of simultaneous inhibition of PI3K/mTOR and ERK pathways in HER2-positive breast cancer instead of the administration of PI3K/mTOR inhibitors used as monotherapy. The inhibition of the ERK pathway can be achieved by either MEK inhibitors or anti-HER2 agents. Clinical trials testing the feasibility of combining PI3K inhibitors with MEK inhibitors are currently being initiated. This combination could be potentially challenging since these two classes of agents may have overlapping toxicities such as skin rash (Shapiro et al., 2009; Banerji et al., 2010). On the other hand, the combination of mTOR allosteric inhibitors and trastuzumab has already been shown to be safe (André et al., 2008) and we have recently started the enrollment of patients with HER2overexpressing breast cancer in a clinical trial combining the PI3K/mTOR inhibitor BEZ235 with the anti-HER2 monoclonal antibody trastuzumab.

\section{Materials and methods}

Cell lines, treatments and siRNA

All the cell lines were obtained from the American Type Culture Collection (Rockville, MD, USA) and maintained in Dulbecco's modified Eagle medium/Ham F12 1:1 (DMEM/ F12) supplemented with $10 \%$ fetal bovine serum and $2 \mathrm{~mm}$ L-glutamine (Life Technologies, Inc. Ltd, Paisley, UK) at $37^{\circ} \mathrm{C}$ in $5 \% \mathrm{CO}_{2}$. MCF7-HER2 cells were obtained as previously described (Scaltriti et al., 2007) and cultured with hygromycin $30 \mu \mathrm{g} / \mathrm{ml}$. BT474-Tr cells were obtained after 1.5 years exposure of BT474 cells to trastuzumab (Scaltriti et al., 2009). BEZ235, NVP-AEW541, the AKT1/2/3 inhibitor, Lapatinib and Torin were obtained elsewhere (see Acknowledgements). The PI3K inhibitors GDC-0941 and PIK-90 were purchased at Axon Medchem (Groningen, The Netherlands). AZD6244 was obtained from Otava Chemicals (Toronto, Ontario, Canada) and erlotinib from Selleck Chemicals (Houston, TX, USA). UO126 and SRC-KI-I were purchased at Cell Signaling (Danvers, MA, USA) and Calbiochem (Darmstadt, Germany), respectively. All the above compounds were dissolved in dimethyl sulfoxide. Trastuzumab was kindly provided by F Hoffmann, La Roche, Basel, Switzerland and was dissolved in sterile apyrogen water.

To knockdown p110- $\alpha$, we used previously published siRNA sequences (Stealth, Invitrogen, Paisley, UK) (Crowder et al., 2009). Transfection was performed with Dharmafect 1 (Dharmacon, Lafayette, CO, USA) at $50 \mathrm{~nm}$ of the siRNAs (scramble and against $\mathrm{p} 110-\alpha$ ) for $6 \mathrm{~h}$ in Optimem media (Invitrogen). The effects of $\mathrm{p} 110-\alpha$ knockdown were evaluated $72 \mathrm{~h}$ after transfection.

Western blot, immunoprecipitation and cross-link

Protein lysates were obtained as previously described (Scaltriti et al., 2009). Briefly, total lysates were resolved by sodium dodecyl sulfate polyacrylamide gel electrophoresis and electrophoretically transferred to nitrocellulose membranes. Membranes were hybridized with the following primary antibodies from Cell Signaling: P-EGFR-Tyr1068, P-HER2-Tyr1221/2,

\section{References}

André F, Campone M, Hurvitz SA, Vittori L, Pylvaenaeinen I, Sahmoud T et al. (2008). Multicenter phase I clinical trial of daily and weekly RAD001 in combination with weekly paclitaxel and
P-HER3-Tyr1289, P-Akt-Ser473, P-Akt-Thr308, Akt, P-ERKThr202/Tyr204, ERK, P-S6-Ser240/244 in 5\% BSA or with HER2 (BioGenex, Fremont, CA, USA), HER3 (NeoMarkers, Fremont, CA, USA), EGFR (Sigma, St Louis, MO, USA or Santa Cruz, Santa Cruz, CA, USA for BT474) in 5\% non-fat dry milk. Mouse and rabbit HRP-conjugated secondary antibodies (Amersham Biosciences, Uppsala, Sweden) were used at 1:3000 in phosphate buffered saline (PBS)-T/2\% nonfat dry milk. Protein-antibody complexes were detected by chemiluminescence with the SuperSignal West Dura Extended Duration Substrate (Pierce, IL, USA), and images were captured with a FUJIFILM LAS-3000 camera system (Fujifilm, Tokyo, Japan). The experiments were repeated at least three times.

For immunoprecipitation experiments, volumes of $500 \mu \mathrm{l}$ of lysis buffer containing equal amount of protein were incubated with $10 \mu \mathrm{g}$ of trastuzumab or $4 \mu \mathrm{g}$ of $\mathrm{p} 85-\mathrm{N}-\mathrm{SH} 2$ domain antibody (Millipore, Billerica, MA, USA, 06-496) overnight at $4{ }^{\circ} \mathrm{C}$ with gentle rotation. Protein A sepharose beads (Amersham Biosciences) were added for $2 \mathrm{~h}$ and washed three times with lysis buffer before suspension in sodium dodecyl sulfateloading buffer.

For cross-linking experiments, cells were detached using $10 \mathrm{~mm}$ ethylenediaminetetraacetic acid (EDTA) in PBS and gentle scraping, and incubated in $5 \mathrm{~mm}$ bis(sulfosuccinimidyl) suberate (BS3) for $30 \mathrm{~min}$ at room temperature with gentle rotation. Cross-linking reaction was stopped by incubating cells in $25 \mathrm{~mm}$ Tris- $\mathrm{HCl}$ for $15 \mathrm{~min}$ at room temperature with gentle rotation. Cells were then processed for immunoprecipitation with trastuzumab as described above.

Please see Supplementary Materials and methods for the description of the phosphotyrosine arrays, quantitative real-time polymerase chain reaction (qRT-PCR), immunocytofluorescence, proliferation and apoptosis assays, tumor xenografts in nude mice, immunohistochemistry and immunofluorescence, channel enhanced enzyme reaction immunoassay and statistical analysis

\section{Conflict of interest}

José Baselga and Neal Rosen are consultants and advisors for Novartis, Merck and Roche. Sarat Chandarlapaty is consultant for Merck. Sharat Singh is an employee of Prometheus Therapeutics and Diagnostics.

\section{Acknowledgements}

This research was supported by grants from the Instituto de Salud Carlos III (Intrasalud PSO9/00623), from the Breast Cancer Research Foundation and from the ERC (AdG09 250244). BEZ235 and NVP-AEW541 were kindly provided by C Garcia-Echeverria from Novartis, Basel, Switzerland. The AKT1/2/3 inhibitor was provided by Pradip Majumder at Merck Research Laboratories, Boston. Lapatinib was kindly provided by GlaxoSmithKline, Research Triangle Park, NJ. Torin1 was obtained from Whitehead/DFCI. Trastuzumab was kindly provided by F Hoffmann-La Roche, Basel, Switzerland. trastuzumab in patients with HER2-overexpressing metastatic breast cancer with prior resistance to trastuzumab. J Clin Oncol 26(Suppl); abstr 1003 . 
Banerji U, Camidge DR, Verheul HM, Agarwal R, Sarker D, Kaye SB et al. (2010). The first-in-human study of the hydrogen sulfate (Hyd-sulfate) capsule of the MEK1/2 inhibitor AZD6244 (ARRY142886): a phase I open-label multicenter trial in patients with advanced cancer. Clin Cancer Res 16: 1613-1623.

Baselga J, Semiglazov V, van Dam P, Manikhas A, Bellet M, Mayordomo J et al. (2009). Phase II randomized study of neoadjuvant everolimus plus letrozole compared with placebo plus letrozole in patients with estrogen receptor-positive breast cancer. $J$ Clin Oncol 27: 2630-2637.

Baselga J, Swain SM. (2009). Novel anticancer targets: revisiting ERBB2 and discovering ERBB3. Nat Rev Cancer 9: 463-475.

Bellacosa A, de Feo D, Godwin AK, Bell DW, Cheng JQ, Altomare DA et al. (1995). Molecular alterations of the AKT2 oncogene in ovarian and breast carcinomas. Int J Cancer 64: 280-285.

Berns K, Horlings HM, Hennessy BT, Madiredjo M, Hijmans EM, Beelen K et al. (2007). A functional genetic approach identifies the PI3K pathway as a major determinant of trastuzumab resistance in breast cancer. Cancer Cell 12: 395-402.

Brunet A, Bonni A, Zigmond MJ, Lin MZ, Juo P, Hu LS et al. (1999). Akt promotes cell survival by phosphorylating and inhibiting a Forkhead transcription factor. Cell 96: 857-868.

Cantley LC. (2002). The phosphoinositide 3-kinase pathway. Science 296: $1655-1657$.

Carpten JD, Faber AL, Horn C, Donoho GP, Briggs SL, Robbins CM et al. (2007). A transforming mutation in the pleckstrin homology domain of AKT1 in cancer. Nature 448: 439-444.

Carracedo A, Ma L, Teruya-Feldstein J, Rojo F, Salmena L, Alimonti A et al. (2008). Inhibition of mTORC1 leads to MAPK pathway activation through a PI3K-dependent feedback loop in human cancer. J Clin Invest 118: 3065-3074.

Chan S, Scheulen ME, Johnston S, Mross K, Cardoso F, Dittrich C et al. (2005). Phase II study of temsirolimus (CCI-779), a novel inhibitor of mTOR, in heavily pretreated patients with locally advanced or metastatic breast cancer. J Clin Oncol 23: 5314-5322.

Chandarlapaty S, Sawai A, Scaltriti M, Rodrik-Outmezguine V, Grbovic-Huezo O, Serra V et al. (2011). AKT inhibition relieves feedback suppression of receptor tyrosine kinase expression and activity. Cancer Cell (doi:10.1016/j.ccr.2010.10.031).

Chaturvedi D, Gao X, Cohen MS, Taunton J, Patel TB. (2009). Rapamycin induces transactivation of the EGFR and increases cell survival. Oncogene 28: 1187-1196.

Citri A, Yarden Y. (2006). EGF-ERBB signalling: towards the systems level. Nat Rev Mol Cell Biol 7: 505-516.

Clark AS, West K, Streicher S, Dennis PA. (2002). Constitutive and inducible Akt activity promotes resistance to chemotherapy, trastuzumab, or tamoxifen in breast cancer cells. Mol Cancer Ther 1: $707-717$.

Cloughesy TF, Yoshimoto K, Nghiemphu P, Brown K, Dang J, Zhu S et al. (2008). Antitumor activity of rapamycin in a Phase I trial for patients with recurrent PTEN-deficient glioblastoma. PLoS Med 5: e8.

Courtney KD, Corcoran RB, Engelman JA. (2010). The PI3K pathway as drug target in human cancer. J Clin Oncol 28 : 1075-1083.

Crowder RJ, Phommaly C, Tao Y, Hoog J, Luo J, Perou CM et al. (2009). PIK3CA and PIK3CB inhibition produce synthetic lethality when combined with estrogen deprivation in estrogen receptorpositive breast cancer. Cancer Res 69: 3955-3962.

Davies H, Bignell GR, Cox C, Stephens P, Edkins S, Clegg S et al. (2002). Mutations of the BRAF gene in human cancer. Nature 417: 949-954.

Eichhorn PJ, Gili M, Scaltriti M, Serra V, Guzman M, Nijkamp W et al. (2008). Phosphatidylinositol 3-kinase hyperactivation results in lapatinib resistance that is reversed by the $\mathrm{mTOR} /$ phosphatidylinositol 3-kinase inhibitor NVP-BEZ235. Cancer Res 68: 9221-9230.

Ellard SL, Clemons M, Gelmon KA, Norris B, Kennecke H, Chia S et al. (2009). Randomized phase II study comparing two schedules of everolimus in patients with recurrent/metastatic breast cancer: NCIC Clinical Trials Group IND. 163. J Clin Oncol 27: 4536-4541.

Engelman JA, Chen L, Tan X, Crosby K, Guimaraes AR, Upadhyay $\mathrm{R}$ et al. (2008). Effective use of PI3K and MEK inhibitors to treat mutant Kras G12D and PIK3CA H1047R murine lung cancers. Nat Med 14: 1351-1356.

Fan QW, Knight ZA, Goldenberg DD, Yu W, Mostov KE, Stokoe D et al. (2006). A dual PI3 kinase/mTOR inhibitor reveals emergent efficacy in glioma. Cancer Cell 9: 341-349.

Flaherty K, Puzanov J, Sosman J, Kim K, Ribas A, McArthur G et al. (2009). Phase I study of PLX4032: Proof of concept for V600E BRAF mutation as a therapeutic target in human cancer. ASCO Annual Meeting. J Clin Oncol 27: 15s (suppl; abstr 9000).

Garcia JM, Silva J, Pena C, Garcia V, Rodriguez R, Cruz MA et al. (2004). Promoter methylation of the PTEN gene is a common molecular change in breast cancer. Genes Chromosomes Cancer 41: $117-124$

Garrett J, Olivares M, Rinehart C, Dave B, Cook R, Chang J et al. (2009). Transcriptional and post-translational upregulation of HER3 (ErbB3) counteracts antitumor effect of HER2 tyrosine kinase inhibitors. Cancer Res 69(24 Suppl): Abstract nr 63.

Guertin DA, Sabatini DM. (2007). Defining the role of mTOR in cancer. Cancer Cell 12: 9-22.

Hoeflich KP, O'Brien C, Boyd Z, Cavet G, Guerrero S, Jung K et al. (2009). In vivo antitumor activity of MEK and phosphatidylinositol 3-kinase inhibitors in basal-like breast cancer models. Clin Cancer Res 15: 4649-4664.

Holbro T, Beerli RR, Maurer F, Koziczak M, Barbas III CF, Hynes NE. (2003). The ErbB2/ErbB3 heterodimer functions as an oncogenic unit: ErbB2 requires ErbB3 to drive breast tumor cell proliferation. Proc Natl Acad Sci USA 100: 8933-8938.

Ihle NT, Lemos Jr R, Wipf P, Yacoub A, Mitchell C, Siwak D et al. (2009). Mutations in the phosphatidylinositol-3-kinase pathway predict for antitumor activity of the inhibitor PX-866 whereas oncogenic Ras is a dominant predictor for resistance. Cancer Res 69: $143-150$.

Kalinsky K, Jacks LM, Heguy A, Patil S, Drobnjak M, Bhanot UK et al. (2009). PIK3CA mutation associates with improved outcome in breast cancer. Clin Cancer Res 15: 5049-5059.

Kolch W. (2000). Meaningful relationships: the regulation of the Ras/Raf/MEK/ERK pathway by protein interactions. Biochem $J$ 351(Part 2): 289-305.

Li J, Yen C, Liaw D, Podsypanina K, Bose S, Wang SI et al. (1997). PTEN, a putative protein tyrosine phosphatase gene mutated in human brain, breast, and prostate cancer. Science $\mathbf{2 7 5}$ : 1943-1947.

Lopez-Knowles E, O’Toole SA, McNeil CM, Millar EK, Qiu MR, Crea $\mathrm{P}$ et al. (2010). PI3K pathway activation in breast cancer is associated with the basal-like phenotype and cancer-specific mortality. Int J Cancer 126: 1121-1131.

Maira SM, Stauffer F, Brueggen J, Furet P, Schnell C, Fritsch C et al. (2008). Identification and characterization of NVP-BEZ235, a new orally available dual phosphatidylinositol 3-kinase/mammalian target of rapamycin inhibitor with potent in vivo antitumor activity. Mol Cancer Ther 7: 1851-1863.

Makhija S, Amler LC, Glenn D, Ueland FR, Gold MA, Dizon DS et al. (2010). Clinical activity of gemcitabine plus pertuzumab in platinum-resistant ovarian cancer, fallopian tube cancer, or primary peritoneal cancer. J Clin Oncol 28: 1215-1223.

Mirzoeva OK, Das D, Heiser LM, Bhattacharya S, Siwak D, Gendelman R et al. (2009). Basal subtype and MAPK/ERK kinase (MEK)-phosphoinositide 3-kinase feedback signaling determine susceptibility of breast cancer cells to MEK inhibition. Cancer Res 69: $565-572$.

Nagata Y, Lan KH, Zhou X, Tan M, Esteva FJ, Sahin AA et al. (2004). PTEN activation contributes to tumor inhibition by trastuzumab, and loss of PTEN predicts trastuzumab resistance in patients. Cancer Cell 6: 117-127. 
O'Reilly KE, Rojo F, She Q-B, Solit D, Mills GB, Smith D et al. (2006). mTOR inhibition induces upstream receptor tyrosine kinase signaling and activates Akt. Cancer Res 66: 1500-1508.

Olayioye MA, Neve RM, Lane HA, Hynes NE. (2000). The ErbB signaling network: receptor heterodimerization in development and cancer. EMBO J 19: 3159-3167.

Saal LH, Holm K, Maurer M, Memeo L, Su T, Wang X et al. (2005). PIK3CA mutations correlate with hormone receptors, node metastasis, and ERBB2, and are mutually exclusive with PTEN loss in human breast carcinoma. Cancer Res 65: 2554-2559.

Saal LH, Johansson P, Holm K, Gruvberger-Saal SK, She QB, Maurer M et al. (2007). Poor prognosis in carcinoma is associated with a gene expression signature of aberrant PTEN tumor suppressor pathway activity. Proc Natl Acad Sci USA 104: 7564-7569.

Scaltriti M, Rojo F, Ocana A, Anido J, Guzman M, Cortes J et al. (2007). Expression of p95HER2, a truncated form of the HER2 receptor, and response to anti-HER2 therapies in breast cancer. J Natl Cancer Inst 99: 628-638.

Scaltriti M, Verma C, Guzman M, Jimenez J, Parra JL, Pedersen K et al. (2009). Lapatinib, a HER2 tyrosine kinase inhibitor, induces stabilization and accumulation of HER2 and potentiates trastuzumab-dependent cell cytotoxicity. Oncogene 28: 803-814.

Serra V, Markman B, Scaltriti M, Eichhorn PJ, Valero V, Guzman M et al. (2008). NVP-BEZ235, a dual PI3K/mTOR inhibitor, prevents PI3K signaling and inhibits the growth of cancer cells with activating PI3K mutations. Cancer Res 68: 8022-8030.

Shapiro G, Kwak E, Baselga J, Rodon J, Scheffold C, Laird AD et al. (2009). ASCO annual meeting. J Clin Oncol 27: 15s (suppl; abstr 3500).

Shi Y, Yan H, Frost P, Gera J, Lichtenstein A. (2005). Mammalian target of rapamycin inhibitors activate the AKT kinase in multiple myeloma cells by up-regulating the insulin-like growth factor

receptor/insulin receptor substrate-1/phosphatidylinositol 3-kinase cascade. Mol Cancer Ther 4: 1533-1540.

Slamon DJ, Clark GM, Wong SG, Levin WJ, Ullrich A, McGuire WL. (1987). Human breast cancer: correlation of relapse and survival with amplification of the HER-2/neu oncogene. Science 235: $177-182$.

Solit DB, Garraway LA, Pratilas CA, Sawai A, Getz G, Basso A et al. (2006). BRAF mutation predicts sensitivity to MEK inhibition. Nature 439: 358-362.

Stemke-Hale K, Gonzalez-Angulo AM, Lluch A, Neve RM, Kuo WL, Davies $\mathrm{M}$ et al. (2008). An integrative genomic and proteomic analysis of PIK3CA, PTEN, and AKT mutations in breast cancer. Cancer Res 68: 6084-6091.

Tabernero J, Rojo F, Calvo E, Burris H, Judson I, Hazell K et al. (2008). Dose- and schedule-dependent inhibition of the mammalian target of rapamycin pathway with everolimus: a phase I tumor pharmacodynamic study in patients with advanced solid tumors. $J$ Clin Oncol 26: 1603-1610.

Vivanco I, Sawyers CL. (2002). The phosphatidylinositol 3-KinaseAkt pathway in human cancer. Nat Rev Cancer 2: 489-501.

Yao E, Zhou W, Lee-Hoeflich ST, Truong T, Haverty PM, EasthamAnderson J et al. (2009). Suppression of HER2/HER3-mediated growth of breast cancer cells with combinations of GDC-0941 PI3K inhibitor, trastuzumab, and pertuzumab. Clin Cancer Res 15: 4147-4156.

Yarden Y, Sliwkowski M. (2001). Untangling the ErbB signalling network. Nat Rev Mol Cell Biol 2: 127-137. OMERIEHTS RESER Works 3.0 Unported License. To view a copy of this license, visit http://creativecommons.org/licenses/by-nc-nd/3.0/

Supplementary Information accompanies the paper on the Oncogene website (http://www.nature.com/onc) 